\title{
Biotechnological Tools to Improve Quality Parameters in Temperate Vegetables
}

\author{
J. Srinivas ${ }^{1 *}$, K. Ravinder Reddy ${ }^{1}$, P. Saidaiah ${ }^{2}$ and A. Umarajashekar ${ }^{3}$ \\ ${ }^{1}$ Department of Horticulture, Vegetable Science, ${ }^{2}$ Department of Genetics and Plant Breeding, \\ SKLTSHU, Rajendranagar, Hyderabad-500030, Telangana, India \\ ${ }^{3}$ Regional Agricultural Research Station, PJTSAU, Polasa, Jagityal, \\ Karimnagar, Telangana, India \\ *Corresponding author email id
}

\section{A B S T R A C T}

Ke y w o r d s
Temperate
vegetables,
Nutritional
improvement,
Shelf life, Lettuce
Natural products.
Article Info
Accepted:
29 May 2017
Available Online:
10 June 2017

\section{Keywords}

Temperate vegetables Nutritional Shelf life, Lettuce

June 2017
Biotechnology has played an important role towards the attainment of production sustainability of crops by using various biotechnological tools. Biotechnology has the potential to provide new opportunities for achieving enhanced crop productivity in a way that will alleviate poverty, improve food security and nutrition, and promote sustainable use of natural resources. Efforts are being made towards nutritional improvement of crops by using the tools of biotechnology by increasing the levels of essential nutrients. A major challenge of sustainable livelihood in the developing nations can be met with the judicious and rational application of biotechnology along with other equally powerful crop production systems. Adoption of biotechnology in agriculture has been the focus of controversy due to varied reasons, as questions have arisen regarding food and environmental safety. Compared with traditional breeding strategies, the application of biotechnology to improve phytonutrient levels in whole foods is more difficult due to the complex array of potentially important chemicals and the complexity of the underlying biosynthetic pathways. To genetic modifications to reduce browning and oxidation and extend the shelf life of prepackaged lettuce. It is a specific plant-produced substances (phytochemicals), as well as classes of phytochemicals that offer specific health benefits. Phytochemical families with clearly beneficial health properties include glucosinolates found in the brassica vegetables including broccoli; carotenoids, anthocyanins and flavonols found in many temperate vegetables. It is also exploring approaches to retard wilting and yellowing in leafy green vegetables. The targeted expression of cytokinins to senescing leaves has shown promise as a route to extending shelf life of leafy vegetables. In addition, most of the transformants with higher zeaxanthin levels showed also increased total carotenoid contents (up to 5.7-fold) and some of them exhibited reduced amounts of lutein. The increase in total carotenoids suggests that the genetic modification affects the regulation of the whole carotenoid biosynthetic pathway in potato tubers. Purple colour potato was developed by expressing anthocyanin pigment by a gene encoding MYB transcription factor. Transgenic potato was developed by expression of 1-SST (Sucrose: sucrose 1-fructosyl transferase) and 1-FFT (fructan: fructan1-fructosyl transferase) Gene from globe artichoke for inulin production. 


\section{Introduction}

Biotechnology has played an important role towards the attainment of production sustainability of crops by using various biotechnological tools. Biotechnology has the potential to provide new opportunities for achieving enhanced crop productivity in a way that will alleviate poverty, improve food security and nutrition, and promote sustainable use of natural resources in hill farming system.

It could be very difficult to improve nutritional deficiency exclusively by traditional breeding and management practices of crops. Efforts are being made towards nutritional improvement of crops by using the tools of biotechnology by increasing the levels of essential nutrients. A major challenge of sustainable livelihood in the developing nations can be met with the judicious and rational application of biotechnology along with other equally powerful crop production systems. Adoption of biotechnology in agriculture has been the focus of controversy due to varied reasons, as questions have arisen regarding food and environmental safety.

The realistic utilization of biotechnology in agriculture has not only brought about profound changes in the crop productivity and quality, but has also opened up newer and hitherto unforeseen potential vistas including engineering novel traits, successful commercial micropropagation, manipulating plant architecture and molecular farming.

The molecular techniques for plant diseases diagnosis and pathogen characterization, marker assisted selection, parasitic diagnosis, development of transgenic crop, and gender friendly agri-entrepreneurship have found a stay in the economic development and prosperity of farmers in the hilly region. In this chapter, we are reviewing the development challenges facing in hill agriculture and try to elucidate how biotechnology can contribute to food security, poverty reduction and sustainable socioeconomic development.

Breeding and biotechnological approaches are currently used to increase the content of specific bioactive components of plants, but the manipulation of plant metabolism is still not easy to address. There is an increasing awareness that multiple genetic and environmental factors affect production and accumulation of bioactive compounds, but these factors are rarely taken into account when fruit is marketed. Rigorous and unprejudiced evaluation of scientific evidence requires a defined set of criteria and methods of evaluation, particularly when breeding and biotech programs are aimed of producing new varieties with improved nutritional values combined with high plant production efficiency and fruit quality. In order to develop new genotypes and commercial cultivars the availability of new sources of Quality Attributes (QA) and Nutritional Attributes (NA) should be explored.

In the strawberry, wild species such as $F$. virginiana glauca and $F$. vesca are good sources of bioactive compounds, but in raspberries the introduction of the wild germplasm ( $R$. parvifolium) did not improve the nutritional quality of fruit.

The methods available for detecting fruit, Total antioxidant capacity (TAC), combined with Tissue printing hybridization (TPH) and other quality parameters such as sugars, total acidity and fruit color, can be proposed as excellent tools for developing a fast and reliable program for screening large breeding populations for high nutritional quality genotypes. Furthermore, NA can represent a useful tool to facilitate analysis of "substantial 
equivalence" of transgenic and control derived fruit.

Plant biotechnology has the potential to address various problems in agriculture and horticulture.

Biotechnological tools are being employed to minimize yield losses due to various stresses (biotic and abiotic) and are being used extensively for value addition in food crops by enrichment with quality proteins, vitamins, iron, zinc, carotenoids, anthocyanins and so on. Other ongoing efforts include the enhancement of shelf life of fruits and vegetables so as significantly to reduce the post-harvest losses of perishable crops.

\section{Quality improvements}

Plants are remarkable in their capacity to synthesize a variety of organic substances, such as vitamins, sugars, starches and amino acids. As many as 80,000 different substances are synthesized in plants, including macronutrients and micronutrients significant to human health (Dellapenna, 1999). Many of these substances, however, are undesirable "anti-nutrients" which can be detrimental to human health.

The genomics will bolster plant biochemistry as researchers seek to understand the metabolic pathways for the synthesis of these compounds. Identifying rate-limiting steps in synthesis could provide targets for genetically engineering biochemical pathways to produce augmented amounts of compounds and new compounds. Targeted expression will be used to channel metabolic flow into new pathways, while gene-silencing tools will reduce or eliminate undesirable compounds or traits.

\section{Carbohydrates}

Plants manufacture both polymeric carbohydrates, like starch, and individual sugars, like sucrose. Plant starch is used in a wide range of industrial applications such as coatings for paper and textiles and as a gelling agent in the food industry (Heyer et al., 1999). It is now possible to make starches that are free of the amylose fraction, resulting in a gelling agent that is clearer and forms a gel at a lower temperature. Starches with higher levels of amylose are more desirable as coating agents and maintain texture at higher temperatures. For example, "sticky rice" has lower amylose content. The presence or absence of amylose greatly influences the physiochemical properties of starch; genetic engineering will result in specialized starches with higher value for specific applications.

In the paper industry, starches sometimes must be chemically modified by phosphorylation, in which phosphate monoester residues are chemically added to corn starch. Some starches (e.g., potato) are naturally phosphorylated, containing the enzymes to add this residue to the starch molecule. Cloning the gene responsible for phosphorylation of glucans makes it possible to engineer precise levels of phosphorylation, resulting corn starch that is more useful to the paper industry.

The chain length and distribution in amylopectin, the more highly structured (branched) component of starch, can be manipulated by altering the starch synthase activity. Genes responsible for the synthesis of fructans can modify plants of agronomic value to produce this polymeric carbohydrate (Heyer et al., 1999).

Fructans are an important ingredient in "functional" foods because they promote health and help to reduce the risk of colon cancer. Sugar beets that accumulate higher levels of fructans could be developed as a source for low-calorie sweeteners.

The ability to synthesize high molecular weight inulin was transferred to potato plants 
via constitutive expression of the 1-SST (sucrose: sucrose 1-fructosyltransferase) and the 1-FFT (fructan: fructan 1fructosyltransferase) genes of globe artichoke (Cynara scolymus). The fructan pattern of tubers from transgenic potato plants represents the full spectrum of inulin molecules present in artichoke roots as shown by high-performance anion exchange chromatography, as well as size exclusion chromatography. These results demonstrate in plant that the enzymes sucrose: sucrose 1fructosyltransferase and fructan: fructan 1fructosyltransferase are sufficient to synthesize inulin molecules of all chain lengths naturally occurring in a given plant species. Inulin made up 5\% of the dry weight of transgenic tubers, and a low level of fructan production also was observed in fully expanded leaves. Although inulin accumulation did not influence the sucrose concentration in leaves or tubers, a reduction in starch content occurred in transgenic tubers, indicating that inulin synthesis did not increase the storage capacity of the tubers (Elke Hellwege et al., 2000).

\section{Proteins}

Plant proteins provide amino acids important for human health. Many plant proteins, like those present in corn seed, do not have the complete complement of essential amino acids. Plant-seed proteins can be modified to express proteins with a more desirable aminoacid composition.

This is particularly important for animal feeds, where seeds engineered to produce a higher concentration of sulfur-containing amino acids could improve wool growth in sheep. The amino-acid composition of seedstorage proteins found in seeds, nuts, fruits and tubers can be modified to enhance nutritional value. Plants may also be modified to produce proteins that aid in mineral nutrition, such as hemoglobin to improve iron uptake and other specific proteins to improve calcium uptake.

Natalia Gutierrez et al., (2008) Faba beans (Vicia faba L.) have a great potential as a protein-rich fodder crop, but anti-nutritional factors such as condensed tannins reduce the biological value of their protein. Tannins can be removed from seeds by any of the two complementary genes, zt- 1 and zt-2, which also determine white-flowered plants (Fig. 1).

The less common gene, $\mathrm{zt}-2$, is also associated with increased protein levels and energy values and reduced fibre content of the seeds. To identify a cost-effective marker linked to zt-2, we analysed a segregating F2 population derived from the cross between the coloured flower and high tannin content genotype Vf6 and a zt-2 line. By using Bulked Segregant Analysis (BSA), five RAPD markers linked in coupling and repulsion phase to zt-2 were identified and their conversion into Sequence Characterised Amplified Regions (SCARs) was attempted. Amplification of the SCARS was more consistent, although the initial polymorphism was lost. To improve the efficiency of the marker screening, a multiplex PCR was developed that allowed the simultaneous amplification of the SCAR with the same advantages as a codominant marker. Marker validation was carried out with a new F2 population segregating for flower colour and tannin content, under scoring the potential of these markers in breeding selection to introgress the zt-2 gene for the development of new tannin free faba bean cultivars.

\section{Nutritional components}

Health-conscious consumers are compelling farmers and seed companies to improve the overall nutritional quality of their products. Extensive medical, biochemical and 
epidemiological research points to specific plant-produced substances (phytochemicals), as well as classes of phytochemicals that offer specific health benefits. Fruits and vegetables are a major source of beneficial phytochemicals (Dellapenna, 1999).

Phytochemical families with clearly beneficial health properties include glucosinolates found in the brassica vegetables including broccoli; carotenoids, such as the tomato fruit pigment lycopene, found in many plant families; flavonoids, such as the isoflavones found in soybeans; and the anthocyanins and flavonols found in many fruits and vegetables.

Some foods containing consistently higher levels of these and other plant nutrients should be available through conventional breeding methods within 10 years. The natural variation that would provide the basis of health-enhanced varieties may be present already in breeding populations. Compared with traditional breeding strategies, the application of biotechnology to improve phytonutrient levels in whole foods is more difficult due to the complex array of potentially important chemicals and the complexity of the underlying biosynthetic pathways (Forkmann, 1991).

Subhra et al., (2000). Improvement of nutritive value of crop plants, in particular the amino acid composition, has been a major long-term goal of plant breeding programs. Toward this end, we reported earlier the cloning of the seed albumin gene AmA1 from Amaranthus hypochondriacus. The AmA1 protein is nonallergenic in nature and is rich in all essential amino acids, and the composition corresponds well with the World Health Organization standards for optimal human nutrition. In an attempt to improve the nutritional value of potato, the AmA1 coding sequence was successfully introduced and expressed in tuber-specific and constitutive manner.

Fig.1 Reduction of anti-nutritional compounds

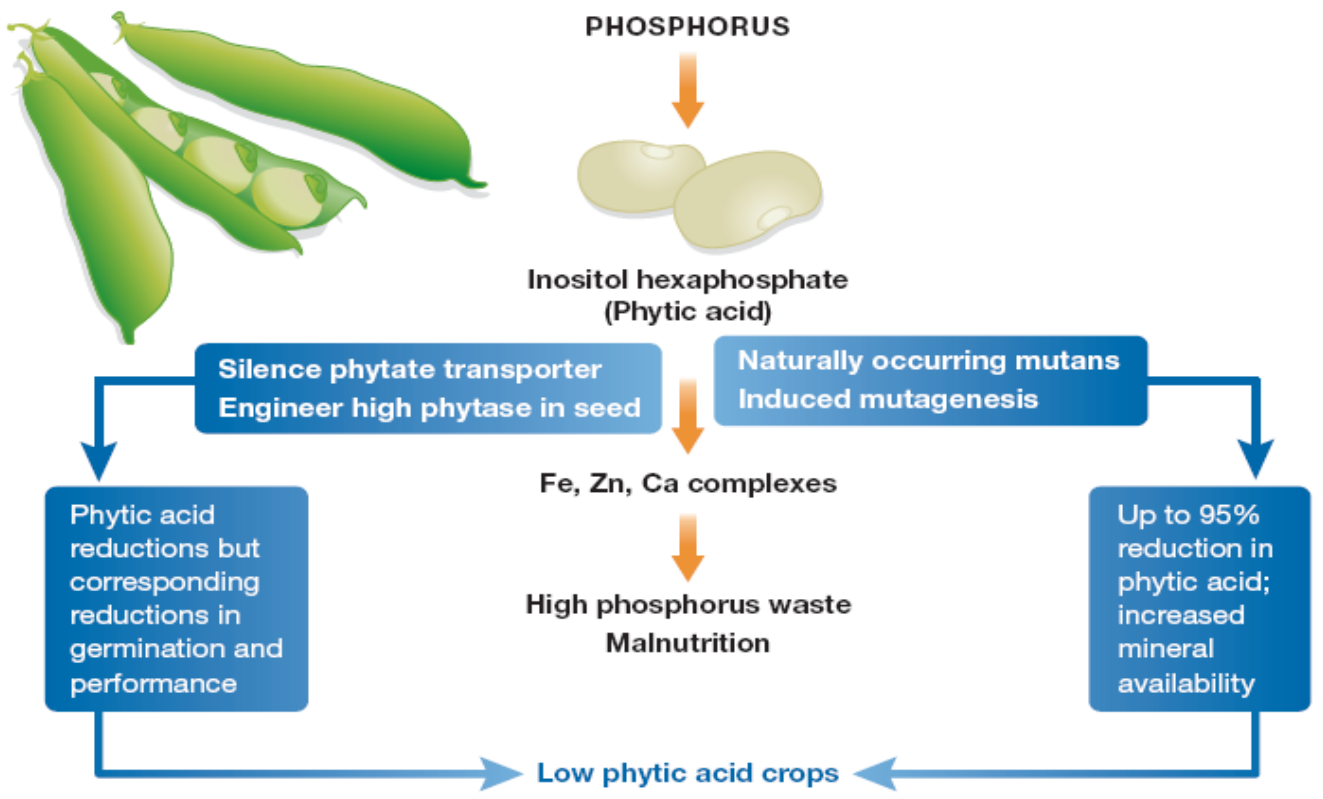


Fig.2 White colour

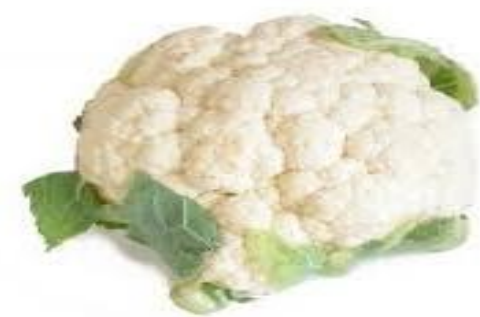

There was a striking increase in the growth and production of tubers in transgenic populations and also of the total protein content with an increase in most essential amino acids. The expressed protein was localized in the cytoplasm as well as in the vacuole of transgenic tubers. Thus we have been able to use a seed albumin gene with a well-balanced amino acid composition as a donor protein to develop a transgenic crop plant. The results document, in addition to successful nutritional improvement of potato tubers, the feasibility of genetically modifying other crop plants with novel seed protein composition.

\section{Flavour and colour}

The ability to transgenically manipulate color intensity and hue was demonstrated more than 10 years ago (Meyer et al., 1987; Napoli et al., 1990). In flowers, the altered expression of the enzymes of flavonoid biosynthesis yielded novel floral pigmentation patterns. Such approaches have not been applied to fruits yet, but the potential exists.

Anthocyanins are the pigments responsible for color in many fruits, such as grapes and strawberries. Deeply colored fruits are generally more desirable to consumers. Further, anthocyanins and related flavonoids have antioxidant properties that reduce the risk of cardiovascular disease and cancer. Fruits with consistently higher levels of anthocyanins, produced through genetic
Fig.3 Orange colour

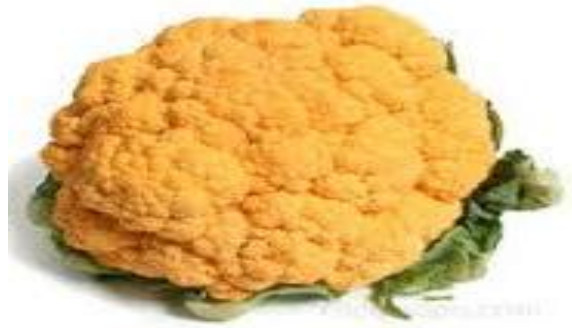

modification, could reach the supermarket within 15 years. These will likely be produced by altering the expression of whole biochemical pathways rather than through modulation of specific enzymes.

Improved flavor is of major interest to consumers, but it does not receive significant attention from breeders, who work largely to improve production and durability during postharvest distribution. The complexity of flavour which includes a balance between sweetness and acidity as well as the compounds that give products their characteristic taste has discouraged the pursuit of biotechnological approaches to flavor improvement.

Biotechnological efforts to improve sweetness have met with little success so far. In some cases, an increase in sweetness leads to a decrease in size that is unacceptable in the market place. In addition, attempts to increase sweetness by expressing nonsugar, sweetnessenhancing proteins such as monellin have been frustrated because their compounds bind to cellular proteins and are subsequently not available to the sensory system.

Transformation of $\mathrm{Or}$ gene into wild type cauliflower converts the white colour of curd into distinct orange colour with increased levels of $\beta$-carotene (Figs. 2 and 3). Examination of the cytological effects of the Or transforents revealed that expression of the $\mathrm{Or}$ transgene lead to the formation large 
membranous chromoplast in cauliflower curd cells of the or transformants (Li et al., 2006).

\section{Texture}

Enzymes: The biocatalysts are responsible for all the biochemical processes of life are used in applications such as bakery and cheese making to improve texture.

\section{Fibre quality}

In cotton plants, fiber cells manufacture individual fibers. Plant transformation will make it possible to deliver and express unique genes within these specialty cells to create unique cotton fiber products. Fiber strength can be improved to make cotton stronger and more lightweight.

Genes that express a variety of different colors could be introduced to provide a greater range of naturally colored fibers. It should also be possible to enhance the quality of cotton fiber by synthesizing other polymeric materials to increase its strength, length and durability. The synthesis of unique polymeric materials in cotton could also enhance its thermal and elastic properties, creating a range of specialty fibers (Maliyakal and Greg 1996).

\section{Shelf life and ripening}

One by Calgene reduces polygalacturonase activity to retard softening, while the other from DNA Plant Technology blocks ethylene synthesis to retard overall ripening. These products were not successful due to limitations in the quality of the base germplasm, the development of competitive nontransgenic products and the difficulty of obtaining premium prices when shelf life is not a primary consumer concern.

Nonetheless, other products with enhanced shelf life should reach the marketplace by the end of this decade. The increased popularity of ready-to-eat and convenience foods will drive the need for products with improved shelf life. For example, sales of prepackaged lettuce have increased over the past 5 years. California agriculture is likely to take advantage of genetic modifications to reduce browning and oxidation and extend the shelf life of prepackaged lettuce. Researchers are also exploring approaches to retard wilting and yellowing in leafy green vegetables. The targeted expression of cytokinins to senescing leaves has shown promise as a route to extending shelf life of leafy vegetables (Ori et al., 1999).

\section{Biotechnology for bio-processing and value addition}

A significant amount of the food produced is lost after harvest thereby aggravating hunger. Most of the temperate vegetables are perishable crops with a lot of storage and post-harvest problems. Productivity is limited by their rapid rate of deterioration soon after harvesting, if processing into more shelfstable products is delayed. Estimates of postharvest losses are hard to determine, but some authorities put losses to as high as 50 percent of what is produced. It is therefore, important to develop and deploy crop varieties with improved shelf life.

Biotechnology can also be used to convert agricultural waste into useful products such as industrial fibres, bioethanol, wine, beers, animal feeds, fertilizers, cosmetics and pharmaceuticals.

We should use biotechnology to generate new technologies and up-scale known and proven technologies for value addition, reducing and eliminating post-harvest losses in the hills. Technologies for processing and value addition are available but not yet commercially transferred to even progressive farmers. 
In conclusion nutritional compounds are normal in temperate vegetables. Quality improvements will increase shelf life as well as increase nutrition in vegetables. Increase with low uptake of vegetable the required quantity of nutritional compounds will be observed by humans being. Many biotechnological approaches are now in research process for the development of nutritionally important characters.

\section{References}

Cecil Stushnoff, Laurence. J, Ducreux. M, Robert D, Hancock. P, Hedley. E, David and Holm, G. 2010. Flavonoid profiling and transcriptome analysis reveals new gene-metabolite correlations in tubers (Solanum tuberosum L.) Journal of Experimental Botany. 61 (4): 1225-1238.

Dellapenna, D. 1999. Nutritional genomics: Manipulating plant micronutrients to improve human health Science. 285:9. DOI: 10.1126 / science.285.5426.375.

Elke M. Hellwege, Sylvia Czapla, Anuschka Jahnke, Lothar Willmitzer and Arnd G. Heyer 2002. Transgenic potato (Solanum tuberosum) tubers synthesize the full spectrum of inulin molecules naturally occurring in globe artichoke (Cynara scolymus) roots. PNAS, 8699-8704.

Forkmann, G. 1991. Flavonoids as flower pigments: The formation of the natural spectrum and its extension by genetic engineering. Plant Breeding. 106: 26.

Heyer, A.G, Lloyd J. R, Kossmann. J. 1999. Production of modified polymeric carbohydrates. Curr Opin Biotech. 1669 (99).
Li, L., Lu, S., Cosman, K.M., Earle, E.D., Garvin, D.F., and Neill, O. J. 2006. $\beta$ Carotene accumulation induced by the cauliflower $\mathrm{Or}$ gene is not due to an increased capacity of biosynthesis. Phytochemistry 67 1177-1184.

Maliyakal, J. E and Greg, K. 1996. Metabolic pathway engineering in cotton: Biosynthesis of polyhydroxybutyrate in fiber cells. Proceedings of the National Academy of Sciences USA. 1073/pnas.93.23.12768.

Meyer P, Heidmann. I, Forkmann, G, Saedler, H. 1987. A new petunia flower colour generated by transformation of a mutant with a maize gene. Nature.1038 / 330677a.

Natalia Gutierrez, A. C, Avila, C. M, Moreno M. T and Torres. A. M. 2008. Development of SCAR markers linked to $\mathrm{zt}-2$, one of the genes controlling absence of tannins in faba bean Australian Journal of Agricultural Research, 59, 62-68.

Ori, N, Juarez. M. T and Jackson. D. 1999. Leaf senescence is delayed in tobacco plants expressing the maize homeobox gene knotted 1 under the control of a senescence- activated promoter. The Plant Cell. 11:80.

Romer, S, Lübeck. J, Kauder. F, Steiger. S, Adomat. C and Sandmann, G. 2002. Genetic engineering of a zeaxanthin-rich potato by antisense inactivation and cosuppression of carotenoid epoxidation. Metab Eng. Oct; 4 (4):263-72.

Subhra Chakraborty, Niranjan Chakraborty and Asis Datta. 2000. Increased nutritive value of transgenic potato by expressing a nonallergenic seed albumin gene from Amaranthus hypochondriacus 37243729, PNAS. Vol. (97).

\section{How to cite this article:}

Srinivas, J., K. Ravinder Reddy, P. Saidaiah and Umarajashekar, A. 2017. Biotechnological Tools to Improve Quality Parameters in Temperate Vegetables. Int.J.Curr.Microbiol.App.Sci. 6(6): 25492556. doi: https://doi.org/10.20546/ijcmas.2017.606.303 